\title{
Dexmedetomidine Relieves Myocardial Ischemia- Reperfusion Injury and Inhibits Apoptosis in Rats via the Janus Kinase 2/Signal Transducers and Activators of Transcription 3 Signaling Pathway
}

\author{
YUXIA WANG, QUNHUI HE AND M. TIAN* \\ Department of Anesthesiology, Yantai Yuhuangding Hospital, Yantai 264000, Shandong Province, China
}

Wang et al.: Effects of Dexmedetomidine on Myocardial Ischemia-Reperfusion Injury

To evaluate the effects of dexmedetomidine on myocardial ischemia-reperfusion injury in rats and its anti-apoptotic role, as well as the mechanism by which it regulates Janus kinase 2 /signal transducers and activators of transcription 3 signal. Rats were randomly assigned into sham, model, experimental and control groups. The left anterior descending coronary artery was ligated to construct the model of myocardial ischemia-reperfusion. Sham group only received threading without ligation. At $1 \mathrm{~h}$ before operation, $5.0 \mu \mathrm{g} / \mathrm{kg}$ dexmedetomidine and $5.0 \mu \mathrm{g} / \mathrm{kg}$ Janus kinase $2 /$ signal transducers and activators of transcription 3 signaling pathway agonist SC-39100 were intraperitoneally injected into experimental and control groups, respectively, while the same dose of normal saline was injected into sham and model groups. The left ventricular systolic pressure, left ventricular end-diastolic pressure, 1eft ventricular pressure rise $\left(+\mathrm{dP} / \mathrm{dt}_{\max }\right)$ and left ventricular pressure drop $\left(-\mathrm{dP} / \mathrm{dt}_{\text {max }}\right)$ were measured using color Doppler ultrasonography. Terminal deoxynucleotidyl transferase mediated dUTP nick end labeling assay was performed to detect cardiomyocyte apoptosis. The expression levels of phosphorylated Janus kinase 2 and phosphorylated signal transducers and activators of transcription 3 were determined using western blotting. Compared with sham group, model group had significantly decreased left ventricular systolic pressure, $+\mathbf{d P} / \mathbf{d t}_{\max }$ and $-\mathbf{d P} / \mathbf{d t}_{\max }$ and raised left ventricular end-diastolic pressure, cardiomyocyte apoptosis rate and phosphorylated Janus kinase 2 and phosphorylated signal transducers and activators of transcription 3 expressions $(p<0.05)$. Experimental and control groups had significantly higher left ventricular systolic pressure, $+d \mathrm{dP} / \mathrm{dt}_{\text {max }},-\mathbf{d P} / \mathrm{dt}_{\text {max }}$ and phosphorylated Janus kinase 2 and phosphorylated signal transducers and activators of transcription 3 expressions and lower left ventricular end-diastolic pressure and cardiomyocyte rate than those of model group $(p<0.05)$. Dexmedetomidine pretreatment can obviously relieve myocardial ischemia-reperfusion injury and cardiomyocyte apoptosis in rats probably by activating the Janus kinase 2/signal transducers and activators of transcription 3 signaling pathway.

Key words: Dexmedetomidine, myocardial ischemia-reperfusion injury, apoptosis, Janus kinase, signal transducers and activators of transcription 3

When drugs alone are unable to alleviate the symptoms of patients with coronary heart disease and other cardiovascular disease progressing to a certain stage, surgery or interventional therapy is needed, inevitably causing Myocardial Ischemia-Reperfusion Injury (MIRI). MIRI itself can lead to the biochemical, morphologic and structural changes in the heart, so that the patients suffer from arrhythmia, metabolic dysfunction, vascular endothelial dysfunction and other cardiac dysfunctions ${ }^{[1]}$. According to a study ${ }^{[2]}$, cardiomyocytes experience oxidative stress, thereby

*Address for correspondence

E-mail: tm1718@163.com

September-October 2021 leading to Reactive Oxygen Species (ROS) enrichment and the resulting massive cardiomyocyte apoptosis is the most basic pathological change in MIRI. It is urgent for clinicians to alleviate the MIRI caused cardiac dysfunction to a largest extent and lower the death rate

This is an open access article distributed under the terms of the Creative Commons Attribution-NonCommercial-ShareAlike 3.0 License, which allows others to remix, tweak, and build upon the work non-commercially, as long as the author is credited and the new creations are licensed under the identical terms

Accepted 19 October 2021

Revised 12 July 2021

Received 22 February 2020

Indian J Pharm Sci 2021;83(5):982-988 
of patients. Dexmedetomidine is a widely used sedative and analgesic drug in Intensive Care Unit (ICU) and clinical anesthesia. A modern pharmacological study showed that ${ }^{[3]}$ dexmedetomidine, a highly selective alpha-2 ( $\alpha 2)$ adrenergic receptor agonist, also has antianxiety, anti-inflammatory and anti-oxidant effects and can protect organs well. Luo et al. ${ }^{[4]}$ reported that in percutaneous coronary intervention surgery, dexmedetomidine helped patients recover their cardiac function after surgery and relieve MIRI during surgery, which reflect the protective effect of dexmedetomidine on the heart. The cardioprotective mechanism of dexmedetomidine, however, has rarely been reported. The Janus kinase 2/Signal Transducer and Activator of Transcription 3 (JAK2/STAT3) pathway is an important player in the physiological processes such as cell growth, proliferation, apoptosis, differentiation, stress and immunoregulation. According to the study of Leng et al. ${ }^{[5]}$, activating the JAK2/STAT3 pathway obviously improved the symptoms of rats with acute myocardial ischemia. In the present study, the rat model of myocardial ischemia-reperfusion was established to explore the possible mechanism of cardioprotective effect of dexmedetomidine from myocardial injury, cardiomyocyte apoptosis and the JAK2/STAT3 pathway, thereby providing a novel insight into the clinical application of dexmedetomidine.

\section{MATERIALS AND METHODS}

\section{Experimental animals:}

Thirty, 8-10 w old Sprague Dawley rats weighing 220-250 g were used.

\section{Reagents:}

Dexmedetomidine hydrochloride injection (2 $\mathrm{ml}$ : $200 \mu \mathrm{g}, 5$ vials/box, NMP No.: H20090248) was purchased from Jiangsu Hengrui Medicine Co., Ltd., and the specific agonist of the JAK2/STAT3 signaling pathway SC-39100 was provided by Sigma. Hematoxylin-Eosin (HE) staining kit was offered by Shanghai Beyotime Biotechnology Co., Ltd. Phosphorylated JAK2 (p-JAK2) and phosphorylated STAT3 (p-STAT3) antibodies and rabbit antibodies against Glyceraldehyde-3-phosphate dehydrogenase (GAPDH) were obtained from Abcam (USA). Terminal deoxynucleotidyl transferase mediated dUTP nick end labeling (TUNEL) and Western blotting kits were purchased from Rebstock (Germany).

\section{Apparatus:}

Fluorescence microscope was sourced from Nikon (Japan). Gel imaging system was provided by BioRad (USA). $-80^{\circ}$ cryogenic refrigerator was purchased from Wiggens (Germany). Electron microscope was obtained from Mitsubishi (Japan). RM2135 microtome was bought from Leica (Germany).

\section{Establishment of MIRI model:}

After fasting for $12 \mathrm{~h}$ before surgery, the rat was intraperitoneally injected with $3 \mathrm{mg} / 1 \quad 10 \%$ chloral hydrate for anesthesia and fixed on the operating table in a supine position and the skin was prepared, disinfected and connected with electrodes to monitor the rat's cardiogram in real time. According to a previous literature ${ }^{[6]}$, the trachea in the neck was cut open and connected with a small animal ventilator. At the same time, the chest was opened to expose the heart and the left anterior descending coronary artery was ligated with 5-0 suture. After the knot was tightened, the macroscopically visible bluish purple or pale left ventricular wall, decreased blood pressure, slowed fluctuations and obvious elevation of ST segment (interval between ventricular depolarization and repolarization) on the electrocardiogram were regarded as the criteria for successful myocardial ischemia in rats. Following 30 min of ligation, the ligature was cut and reperfusion was performed for $120 \mathrm{~min}$. A more than $1 / 2$ decrease in ST segment on the electrocardiogram indicated successful reperfusion. After modeling, the chest was closed, the wound was sutured and a small amount of penicillin was intraperitoneally injected to prevent infection.

\section{Experimental grouping:}

The rats were randomly divided into sham, model, experimental and control groups $(n=10)$. The rats in sham group only received threading without ligation. At $1 \mathrm{~h}$ before operation, $5.0 \mu \mathrm{g} / \mathrm{kg}$ dexmedetomidine and $5.0 \mu \mathrm{g} / \mathrm{kg}$ JAK2/STAT3 signaling pathway agonist ${ }^{[7]}$ SC-39100 were intraperitoneally injected into the rats in experimental group and control group, respectively, while the same dose of normal saline was injected to those in sham group and model group.

\section{Detection of cardiac function by color Doppler ultrasonography:}

After $120 \mathrm{~min}$ of reperfusion, all groups of rats were anesthetized and then their cardiac functions 
were examined using small animal color Doppler ultrasonography. The Left Ventricular Systolic Pressure (LVSP), Left Ventricular End-Diastolic Pressure (LVEDP), 1eft ventricular pressure rise, (maximal rate of rise of (usually) left ventricular pressure $\left(+\mathrm{dP} / \mathrm{dt}_{\text {max }}\right)$ ) and 1 eft ventricular pressure drop $\left(-\mathrm{dP}_{\mathrm{dt}} \mathrm{dt}_{\max }\right)$ were recorded.

\section{Observation of myocardial tissue injury:}

Following color Doppler ultrasonography, anesthesia was induced for rats and $5 \mathrm{ml}$ of abdominal arterial serum was collected. Finally, the animals were sacrificed. The heart was aseptically stripped and the water was sucked up. The heart was then sealed in liquid nitrogen and placed in the cryogenic refrigerator at $-80^{\circ}$ for later use. A total of $100 \mathrm{mg}$ of myocardial tissues were taken from the rats in all groups, stained by HE and prepared into sections. The pathological changes in the myocardial tissues were observed under the light microscope in each group.

\section{Measurement of serum biochemical indices:}

After centrifugation of blood sample, the serum was retained and the content of Lactate dehydrogenase (LDH), Creatine phosphokinase (CK), Superoxide dismutase (SOD) and Malondialdehyde (MDA) was measured by Enzyme-Linked Immunosorbent Assay (ELISA).

\section{Detection of cardiomyocyte apoptosis by TUNEL assay:}

A total of $100 \mathrm{mg}$ of myocardial tissues were taken from each group, fixed, cryosectioned, dehydrated and embedded, and then they were subjected to TUNEL assay according to the requirements of the TUNEL kit, rinsed, dehydrated and mounted. Finally, the sections were observed under the fluorescence microscope. Normal cardiomyocytes are blue, while apoptotic cells are brown ${ }^{[8]}$. The apoptosis rate of cardiomyocytes in rats in all groups was statistically analyzed and obtained using the Image 8.0 analysis software.

\section{Detection of p-JAK2 and p-STAT3 protein expression levels in myocardial tissues by western blotting:}

A total of $100 \mathrm{mg}$ of myocardial tissues were taken from each group, conventionally lysed, homogenized and centrifuged. Then protein concentration was determined and electrophoresis was performed for separation, followed by transferring and sealing.
Afterwards, the primary antibody (1:500) was added dropwise and incubated at $4^{\circ}$ overnight. On the $2^{\text {nd }}$ $\mathrm{d}$, the resulting proteins were washed and added with the secondary antibody $(1: 1,000)$, followed by color development. The grey value of each band was read using the gel imager and the relative expression level of the target protein was calculated with GAPDH as an internal reference.

\section{Statistical analysis:}

Statistical Package for the Social Sciences (SPSS) 16.0 software was used for statistical analysis and GraphPad Prism 5.01 was adopted for plotting. The $\mathrm{t}$ test was conducted for intergroup comparisons, $\mathrm{p}<0.05$ was considered to be statistically significant.

\section{RESULTS AND DISCUSSION}

Effects of dexmedetomidine pretreatment on cardiac functions of rats with myocardial ischemia-reperfusion were described below. Based on the color Doppler ultrasonographic results of rats in each group (fig. 1), $\mathrm{LVSP},+\mathrm{dP} / \mathrm{dt}_{\text {max }}$ and $-\mathrm{dP} / \mathrm{dt}_{\text {max }}$ were significantly reduced $(p<0.05)$, while LVEDP was significantly increased in model group compared with those in sham group, $(p<0.05)$. In comparison with model group, experimental and control groups exhibited significantly increased LVSP, $+\mathrm{dP} / \mathrm{dt}_{\max }$ and $-\mathrm{dP} / \mathrm{dt}_{\max }$ $(\mathrm{p}<0.05)$ and reduced LVEDP $(\mathrm{p}<0.05)$. No statistically significant differences were found between control and experimental groups $(\mathrm{p}>0.05)$.

Effects of dexmedetomidine pretreatment on injury of rats with myocardial ischemia-reperfusion were given below. The results of HE staining (fig. 2) showed that sham group had normal rat myocardial tissues, neatly arranged cells with complete nucleus structure, rich cytoplasmic contents, no edema and necrosis of the intercellular substance and regularly arranged myocardial fibers. In model group, the structure and morphology of rat cardiomyocytes were obviously damaged, the cells were swollen and disorderly arranged with severely damaged nuclear membrane, evidently constricted nucleus, damaged or dissolved cell membrane and enlarged intercellular substance, inflammatory cells infiltrated, the number of necrotic cells was increased and the myocardial fibers were irregularly arranged, with obvious band-shaped infarction. In experimental group, there were relatively normal structure of cardiomyocytes, slightly swollen interstitium and significantly reduced inflammatory infiltration, clearer cell membrane and smaller number 
of necrotic cells compared with those in model group. In control group, the arrangement of cardiomyocytes and the shape of myocardial fibers were more regular, the intercellular substance was slightly swollen and the cell boundaries were clearer.

Effects of dexmedetomidine pretreatment on serum biochemical indices of rats with myocardial ischemiareperfusion were shown below. According to ELISA results (fig. 3), the activity of serum LDH and CK and the MDA content were significantly higher in model group than those in sham group $(p<0.05)$, while the activity of
SOD was significantly lower in model group than that in sham group $(p<0.05)$. In contrast with model group, experimental group and control group had significantly reduced activity of serum LDH, $\mathrm{CK}(\mathrm{p}<0.05)$ and MDA content $(\mathrm{p}<0.05)$ and significantly elevated activity of SOD $(p<0.05)$. There were no statistically significant differences between control group and experimental group $(\mathrm{p}>0.05)$.

Effects of dexmedetomidine pretreatment on cardiomyocyte apoptosis of rats with myocardial ischemia-reperfusion were shown below. The results
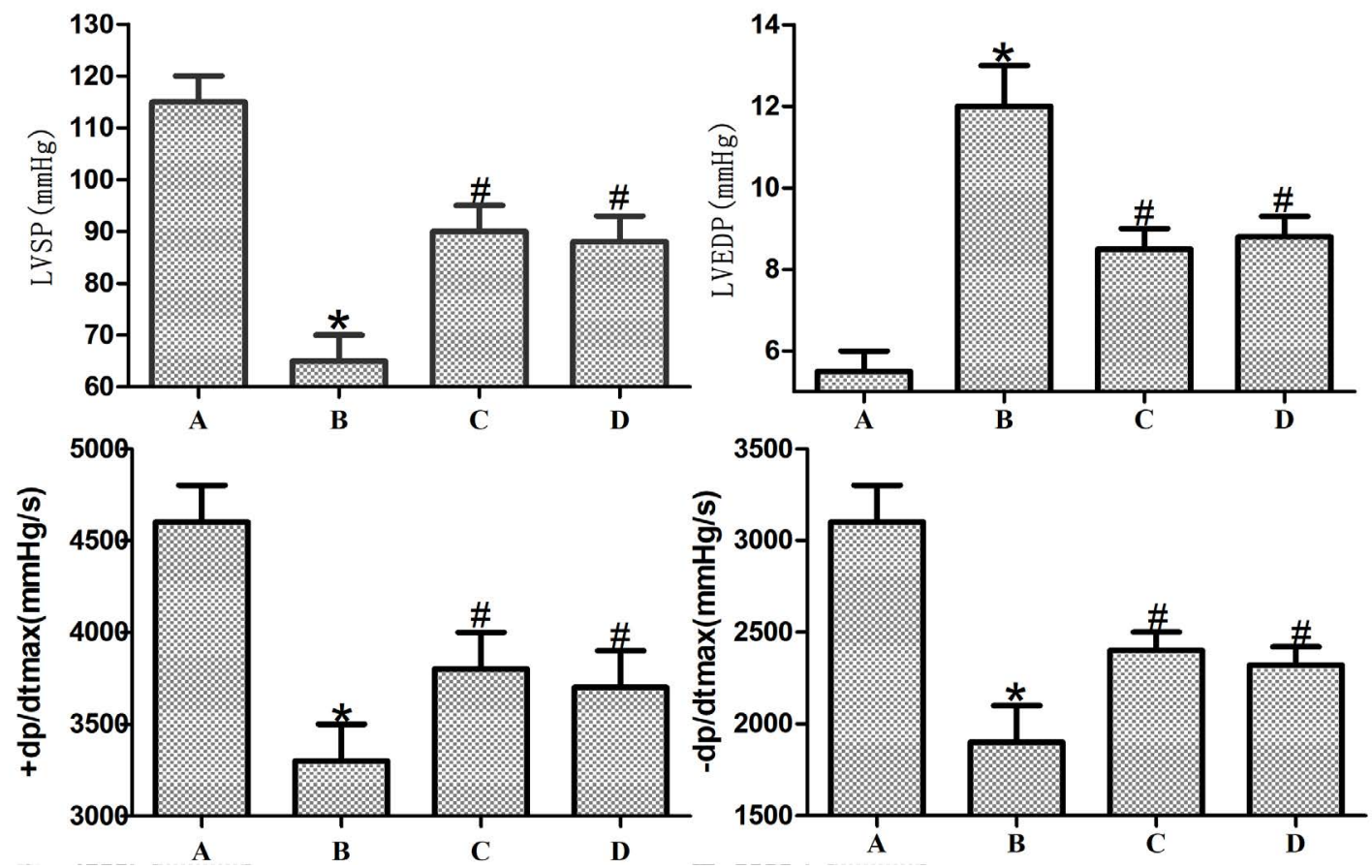

Fig. 1: Cardiac functions of different groups. (A) Sham group; (B) Model group; (C) Experimental group; (D) Control group, ${ }^{*} \mathbf{p}<0.05$ vs. sham group, ${ }^{\#} \mathbf{p}<0.05$ vs. model group
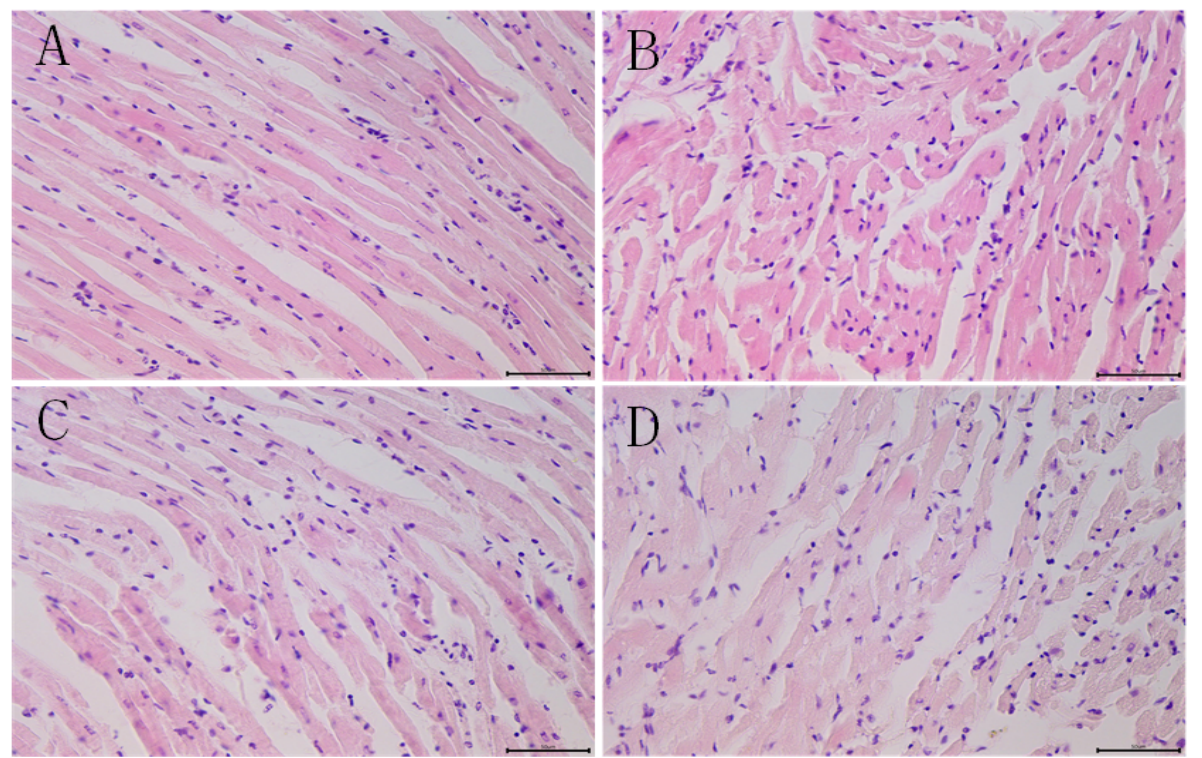

Fig. 2: Pathological changes of myocardial injury in different groups. (A) Sham group; (B) Model group; (C) Experimental group; (D) Control group 
of TUNEL assay (fig. 4) uncovered that the apoptosis rate of cardiomyocytes was obviously higher in model group than that in sham group $(\mathrm{p}<0.05)$ and evidently lower in experimental group and control group than that in model group $(\mathrm{p}<0.05)$ and showed no statistically significant difference between experimental group and control group ( $\mathrm{p}>0.05)$.

Effects of dexmedetomidine pretreatment on JAK2/ STAT3 signaling pathway in rats with myocardial ischemia-reperfusion were given below. Based on the results of western blotting (fig. 5), the p-JAK2 and p-STAT3 expressions in cardiomyocytes were obviously higher in model group than those in sham group $(\mathrm{p}<0.05)$ and also clearly higher in experimental group and control group than those in model group $(p<0.05)$, but they had no statistically significant differences between experimental group and control group ( $\mathrm{p}>0.05)$.

MIRI refers to a pathological condition that myocardial injury is aggravated after reperfusion due to short-term ischemia and hypoxia in the heart during revascularization in patients. MIRI leads to cardiac ultrastructure injury and cardiac dysfunction. Besides, the accumulated ROS during reperfusion is able to induce unexplained necrosis of cardiomyocytes and weakened myocardial systolic function. In particular, perioperative MIRI may lead to severe arrhythmia or even sudden death in patients ${ }^{[9]}$. For this reason, finding
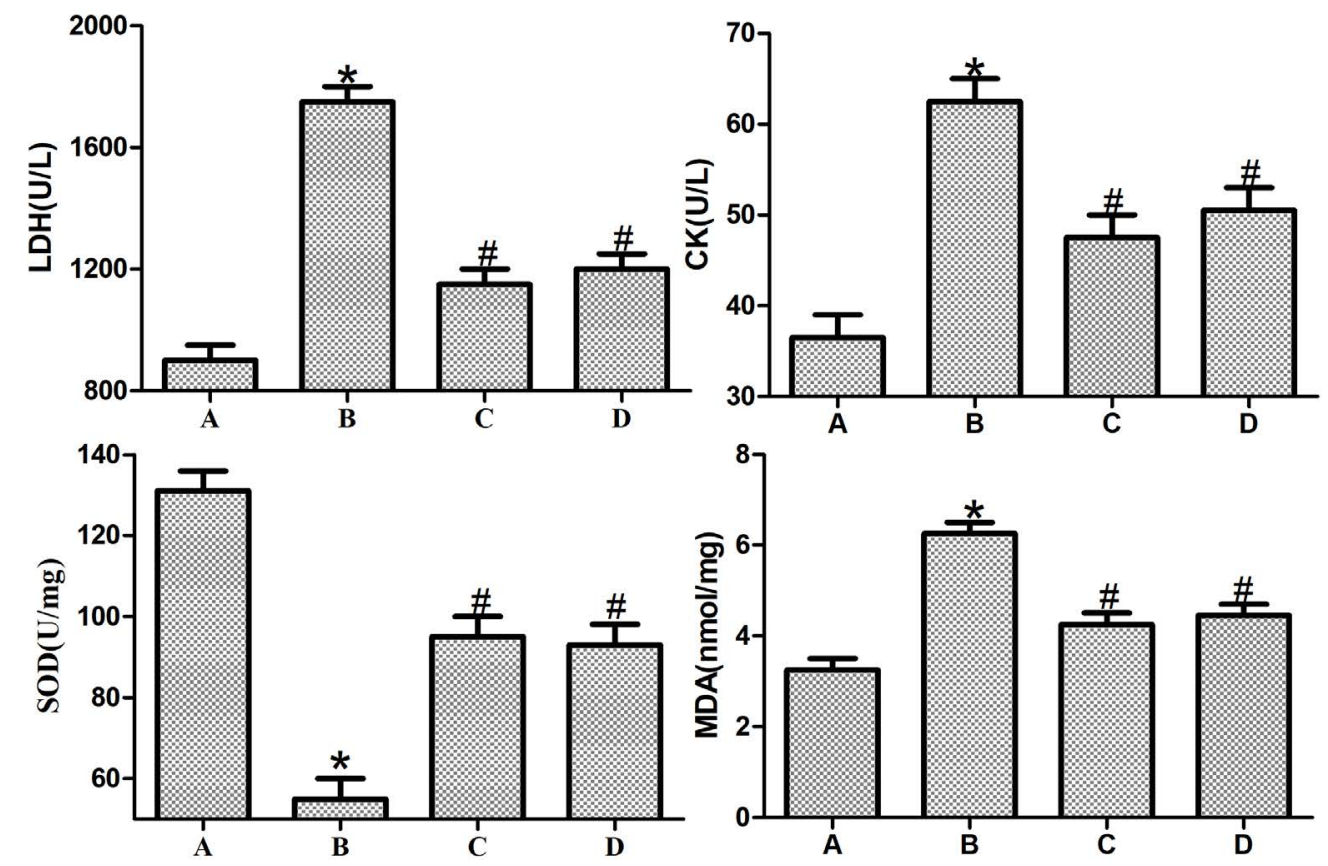

Fig. 3: Serum biochemical indices of different groups. (A) Sham group; (B) Model group; (C) Experimental group; (D) Control group, *p $<0.05$ vs. sham group, ${ }^{\#} \mathbf{p}<0.05$ vs. model group
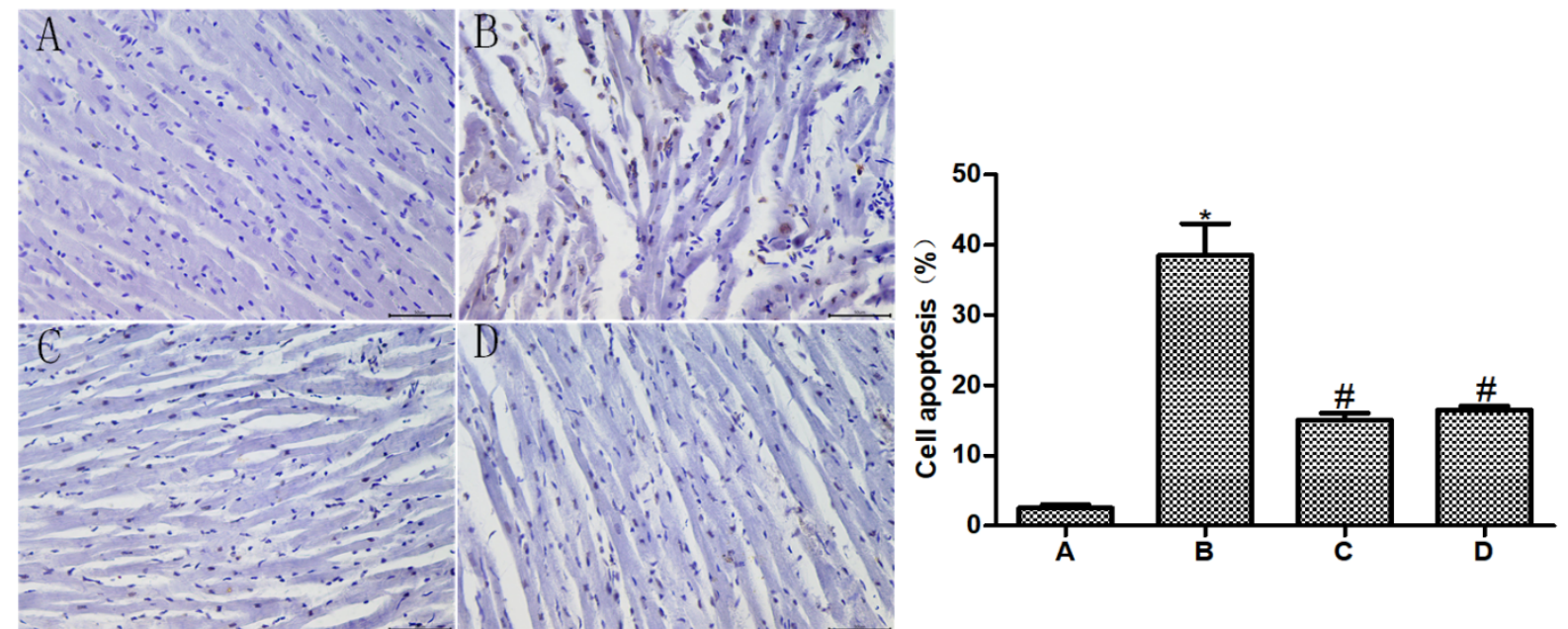

Fig. 4: Apoptosis of cardiomyocytes in different groups. (A) Sham group; (B) Model group; (C) Experimental group; (D) Control group, ${ }^{*} \mathbf{p}<0.05$ vs. sham group, ${ }^{\#} \mathbf{p}<0.05$ vs. model group 


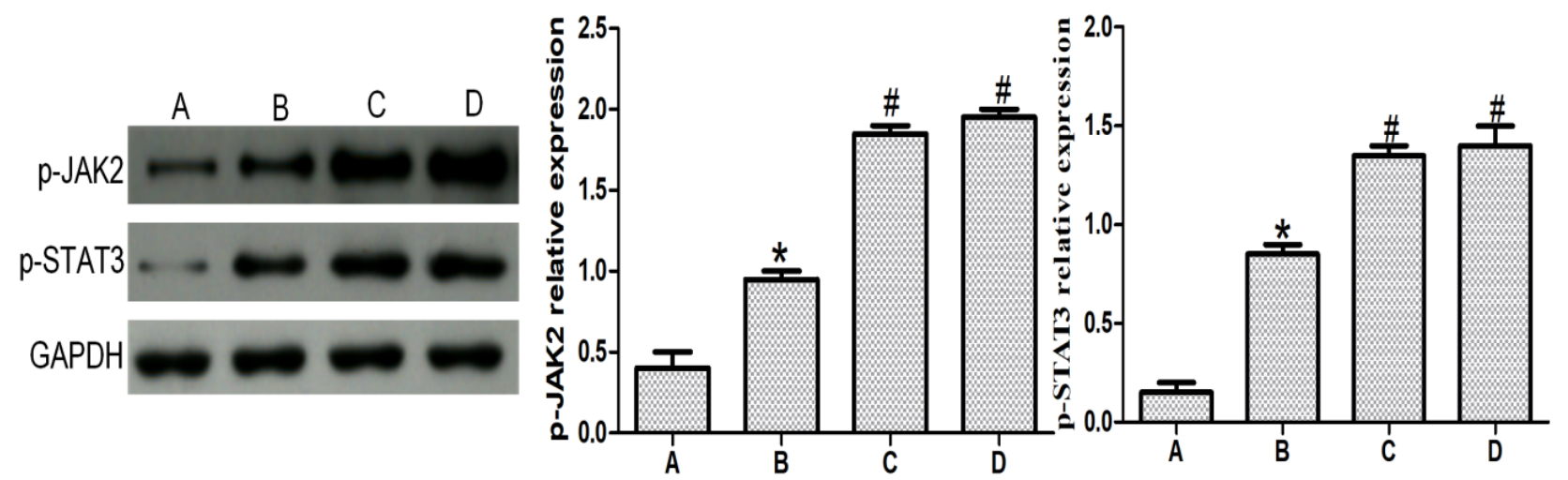

Fig. 5: Protein expressions of p-JAK2 and p-STAT3 in cardiac tissues of different groups. (A) Sham group; (B) Model group; (C) Experimental group; (D) Control group, *p<0.05 vs. sham group, ${ }^{*} p<0.05$ vs. model group

effective means or drugs to inhibit cardiomyocyte apoptosis and reduce MIRI is an important subject to be solved urgently in the treatment of myocardial ischemia.

Studies have manifested that ischemic preconditioning is capable of significantly relieving MIRI, but its clinical application is limited since the absorption and tolerance of different patients to different drugs are varying. To change such a situation, many clinicians have been discovering, researching and screening appropriate drugs. Dexmedetomidine has been approved for sedation and analgesia of patients in ICU by the U.S. Food and Drug Administration as long ago as 1999. Recently, dexmedetomidine has aroused wide attention due to its unique advantages in organ protection during the perioperative period ${ }^{[10]}$. However, the application of dexmedetomidine in myocardial ischemia-reperfusion is rarely reported. In this study, the rat model of myocardial ischemia-reperfusion was established and it was found through color Doppler ultrasonography as well as HE and electron microscopy that model group displayed significantly weakened cardiac function and obvious MIRI. However, the cardiac function was significantly improved and the pathological damage was significantly relieved in experimental group after dexmedetomidine preconditioning. The activity of LDH and $\mathrm{CK}$ has a direct association with the permeability of cardiomyocyte membrane, which is an important factor measuring the structural injury of the heart. The activity of serum LDH and CK in patients is a gold index for clinical diagnosis of such myocardial injuries as angina pectoris and myocardial infarction and also a crucial indicator of the long-term prognosis of cardiovascular patients $^{[11]}$. In this study, the activity of LDH and CK in experimental group was dramatically reduced, implying that dexmedetomidine preconditioning can visibly relieve myocardial injury in rats experiencing myocardial ischemia and reperfusion.
The JAK2/STAT3 signal is a vital transduction pathway involving various pathological processes including inflammation, immunity, apoptosis and oxidative stress in the body. A study uncovered that ${ }^{[12]}$ after the JAK2/STAT3 signal is activated by cells, JAK2 is firstly activated, STAT3 is further phosphorylated and extracellular signals are transferred to the nucleus to regulate the expression of target genes, thus exerting biological effects. Xie et al. ${ }^{[13]}$ studied and confirmed that the activated JAK2/STAT3 signal in MIRI rats was able to obviously enhance the expression of antiapoptotic protein B-cell lymphoma 2 (Bcl-2) and reduce apoptosis. Shang et al. ${ }^{[14]}$ showed that the activation of JAK2/STAT3 signal in cardiomyocytes with anoxiareoxygenation injury could signally suppress cell oxidative stress and reduce the accumulation of ROS. In this study, the JAK2/STAT3 signal agonist was used as control group. In control group, the JAK2/ STAT3 signal was activated by the JAK2/STAT3 signal agonist, promoting the phosphorylation of JAK2 and STAT3 to protect the heart. The results revealed that the pathological signs, serum indexes and other indexes of rats in control group showed no significant differences from those in experiment group. It is suggested that the cardioprotective effect of dexmedetomidine preconditioning may be related to the activation of JAK2/STAT3 signal.

In conclusion, dexmedetomidine preconditioning can significantly reduce MIRI and cardiomyocyte apoptosis in rats, which may have a relation to the activation of JAK2/STAT3 signal. However, further study should be carried out to figure out whether dexmedetomidine preconditioning can alleviate the ischemia-reperfusion injury of patients.

\section{Ethical approval:}

The study was approved by the ethics committee of 
Yantai Yuhuangding Hospital (No:YTWD2021021113).

\section{Author's contributions:}

Yuxia Wang and Qunhui He contributed equally to this work.

\section{Conflicts of interest:}

The authors declared no conflict of interest.

\section{REFERENCES}

1. $\mathrm{Hu} \mathrm{H}$, Zhai C, Qian G, Gu A, Liu J, Ying F, et al. Protective effects of tanshinone IIA on myocardial ischemia reperfusion injury by reducing oxidative stress, HMGB1 expression, and inflammatory reaction. Pharm Biol 2015;53(12):1752-8.

2. Tao H, Nuo M, Min S. Sufentanil protects the rat myocardium against ischemia-reperfusion injury via activation of the ERK1/2 pathway. Cytotechnology 2018;70(1):169-76.

3. Kiski D, Malec E, Schmidt C. Use of dexmedetomidine in pediatric cardiac anesthesia. Curr Opin Anaesthesiol 2019;32(3):334-42.

4. Luo J, Wei L, Xuedong WU, Zhao J. Effect of dexmedetomidine on oxidative stress in patients undergoing percutaneous coronary intervention. J Clin Anesthesiol 2017;33(7):668-70.

5. Leng X, Song N, Zang YA, Li FQ, Gu YL. Role of JAK2/ STAT3 signaling pathway in ginsenoside Re pretreatment in prevention of isoproterenol-induced acute myocardial ischemia. Chinese Pharmacol Bull 2018;34(1):103-7.

6. Shu L, Zhang W, Huang C, Huang G, Su G. Troxerutin protects against myocardial ischemia/reperfusion injury via Pi3k/Akt pathway in rats. Cell Physiol Biochem 2017;44(5):1939-48.

7. Liu J, Zhen P, Li XS, Li SS, Tian Q, Chang YF, et al. JAK2/ STAT3 signaling pathway mediates metabolism and antioxidative stress in chondrocytes of osteoarthritis mice. Chin J Mult Organ Dis Elder 2015;1(7):540-6.

8. Hu S, Cao S, Tong Z, Liu J. FGF21 protects myocardial ischemia-reperfusion injury through reduction of miR-145mediated autophagy. Am J Transl Res 2018;10(11):3677-88.

9. Toldo S, Mauro AG, Cutter Z, Abbate A. Inflammasome, pyroptosis, and cytokines in myocardial ischemia-reperfusion injury. Am J Physiol Heart Circ Physiol 2018;315(6):1553-68.

10. Ren J, Li C, Liu Y, Liu H, Dong Z. Protective effect of dexmedetomidine against myocardial ischemia-reperfusion injury in rabbits 1. Acta Cir Bras 2018;33:22-30.

11. Zhang JJ, Peng K, Zhang J, Meng XW, Ji FH. Dexmedetomidine preconditioning may attenuate myocardial ischemia/reperfusion injury by down-regulating the HMGB1-TLR4-MyD88-NF-кB signaling pathway. PLoS One 2017;12(2):e0172006.

12. Liu S, Yang Y, Song YQ, Geng J, Chen QL. Protective effects of N (2)-L-alanyl-L-glutamine mediated by the JAK2/STAT3 signaling pathway on myocardial ischemia reperfusion. Mol Med Rep 2018;17(4):5102-8.

13. Xie XJ, Fan DM, Xi K, Chen YW, Qi PW, Li QH, et al. Suppression of microRNA-135b-5p protects against myocardial ischemia/reperfusion injury by activating JAK2/ STAT3 signaling pathway in mice during sevoflurane anesthesia. Biosci Rep 2017;37(3):BSR20170186.

14. Shang L, Dong P, Du L, Yang X, Wang H, Li S. SERP1 prevents hypoxia-reoxygenation-induced $\mathrm{H} 9 \mathrm{c} 2$ apoptosis through activating JAK2/STAT3 pathway-dependent attenuation of endoplasmic reticulum stress. Biochem Biophys Res Commun 2019;508(1):256-62. 